\title{
Precursor of Color Superconductivity
}

\author{
M. Kitazawa ${ }^{\mathrm{a}}$, T. Koide ${ }^{\mathrm{b}}$, T. Kunihiro ${ }^{\mathrm{b}}$ and Y. Nemoto ${ }^{\mathrm{c}}$

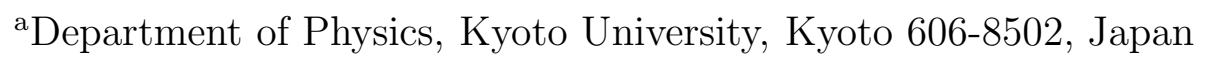 \\ bYukawa Institute for Theoretical Physics, Kyoto University, Kyoto 606-8502, Japan \\ ${ }^{\mathrm{c}}$ RIKEN BNL Research Center, BNL, Upton, NY 11973
}

We investigate possible precursory phenomena of color superconductivity at finite temperature $T$ with an effective theory of QCD. It is found that the fluctuation of the diquark pair field exists with a prominent strength even well above the critical temperature $T_{c}$. We show that such a fluctuaiton forms a collective mode, the corresponding pole of which approaches the origin as $T$ is lowered to $T_{c}$ in the complex energy plane. We discuss the possible relevance of the precursor to the observables to be detected in heavy-ion collisions.

\section{INTRODUCTION}

Determining the phase structure of Quantum Chromodynamics (QCD) is one of the central problems in hadron physics. In particular, at low temperature $(T)$ and non-zero chemical potential $(\mu)$, one may expect that quark matter has a Fermi surface, which gives rise to an interesting complication; if there is some attractive channel in the quark-quark interaction, the Fermi surface becomes unstable and quarks form Cooper pairs, leading to a color superconductivity (CS) with the color-gauge symmetry broken [1].

Since the critical temperature $\left(T_{c}\right)$ of CS is expected to be small, it would be difficult to create the color-superconducting quark matter by heavy-ion collisions. So it is natural that people have exclusively considered neutron stars as a physical system to which CS may have a relevance.

However, there is a possibility to obtain information on CS by heavy-ion collisions through possible fluctuations of the pair field. In fact, it is known that in the ordinary superconductivity in metals, there exist fluctuations of Cooper pairs even above the critical temperature where the pair condensate does not exist: Such fluctuations affect various transport coefficients, which are experimentally observed[2]. In the usual superconductivity in metals, the pairing interaction due to the phonon exchange is so weak that the mean field approximation works well except in the immediate vicinity of the critical point. In other words, the fluctuations become important only near the critical temperature. However, in the case of CS at intermediate densities, large fluctuations may survive even well above $T_{c}$ owing to the strong coupling which may be described by a low-energy effective Lagrangian of QCD. If there are large fluctuations even well above $T_{c}$, heavy-ion collisions on Earth may have a possibility to be utilized to study the physics of CS through 


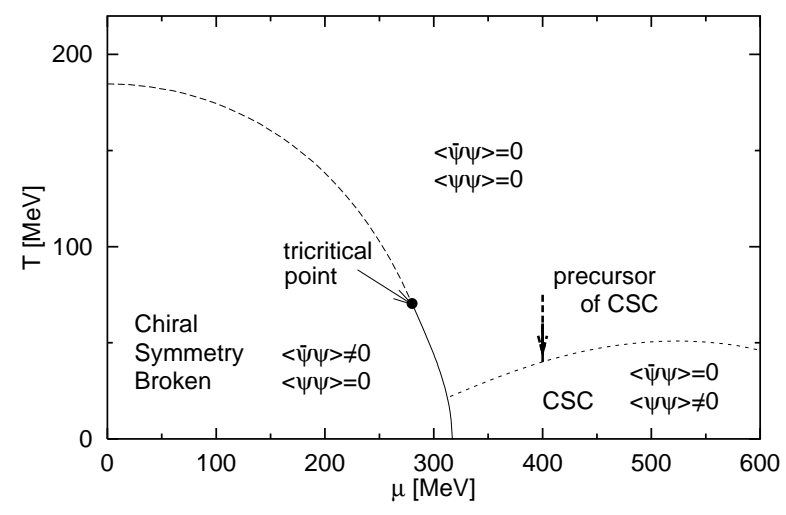

Figure 1. The calculated phase diagram in $T$ $\mu$ plane in our model. The Solid and dashed line denote the critical line of a first and second order phase transition, respectively.

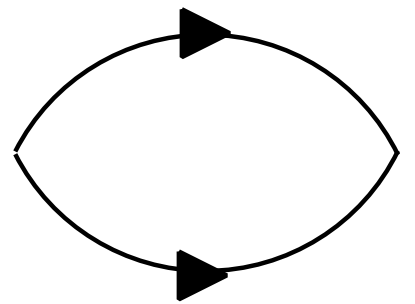

Figure 2. The one loop diagram of the particle-particle correlation.

examining precursory phenomena of CS.

\section{RESPONSE OF PAIR FIELD}

We use the two-flavor Nambu-Jona-Lasinio model in the chiral limit, [ 3]

$\mathcal{L}=\bar{\psi} i \not \partial \psi+G_{S}\left[(\bar{\psi} \psi)^{2}+\left(\bar{\psi} i \gamma_{5} \vec{\tau} \psi\right)^{2}\right]+G_{C}\left(\bar{\psi} i \gamma_{5} \tau_{2} \lambda_{2} \psi{ }^{C}\right)\left(\bar{\psi}^{C} i \gamma_{5} \tau_{2} \lambda_{2} \psi\right)$,

where $\psi^{C} \equiv C \bar{\psi}^{T}$ with $C=i \gamma^{2} \gamma^{0}$ being the charge conjugation operator, and $\tau_{2}$ and $\lambda_{2}$ are the second component of the Pauli and Gell-Mann matrices representing the flavor $\mathrm{SU}(2)$ and color $\mathrm{SU}(3)$, respectively.

The phase structure of our model in the mean field approximation is shown in Fig. 1. The dashed line denotes the critical line of the second order phase transition and the solid line first order phase transition. The tricritical point where the order of the chiral transition changes from the second to first order is located at about $(T, \mu)=(70,280) \mathrm{MeV}$.

Now that the phase structure is determined, we investigate the $T$ dependence of the fluctuation of the diquark pair field in the Wigner phase above $T_{c}$; we calculate the response of the pair field to an external one in the linear response theory. When an external field $\Delta_{e x}^{*}$ is added in the Wigner phase, the induced pair field $\Delta_{\text {ind }}^{*}$ is given by

$\Delta_{i n d}^{*}\left(\mathbf{k}, \omega_{n}\right)=\frac{-G_{C} \mathcal{Q}\left(\mathbf{k}, \omega_{n}\right)}{1+G_{C} \mathcal{Q}\left(\mathbf{k}, \omega_{n}\right)} \Delta_{e x}^{*} \equiv \mathcal{D}\left(\mathbf{k}, i \omega_{n}\right) \Delta_{e x}^{*}$,

where $\mathcal{Q}\left(\mathbf{k}, \omega_{n}\right)$ is a particle-particle correlation function corresponding diagrammatically to Fig. 2. The function $\mathcal{D}\left(\mathbf{k}, i \omega_{n}\right)$ is still a function of imaginary time. By carrying out the analytic continuation, we then obtain, what we call, the response function $D^{R}(\mathbf{k}, \omega)$. Here it should be noticed that the following equality holds

$$
1+\left.G_{C} \mathcal{Q}(\mathbf{0}, 0)\right|_{T=T_{c}}=0
$$



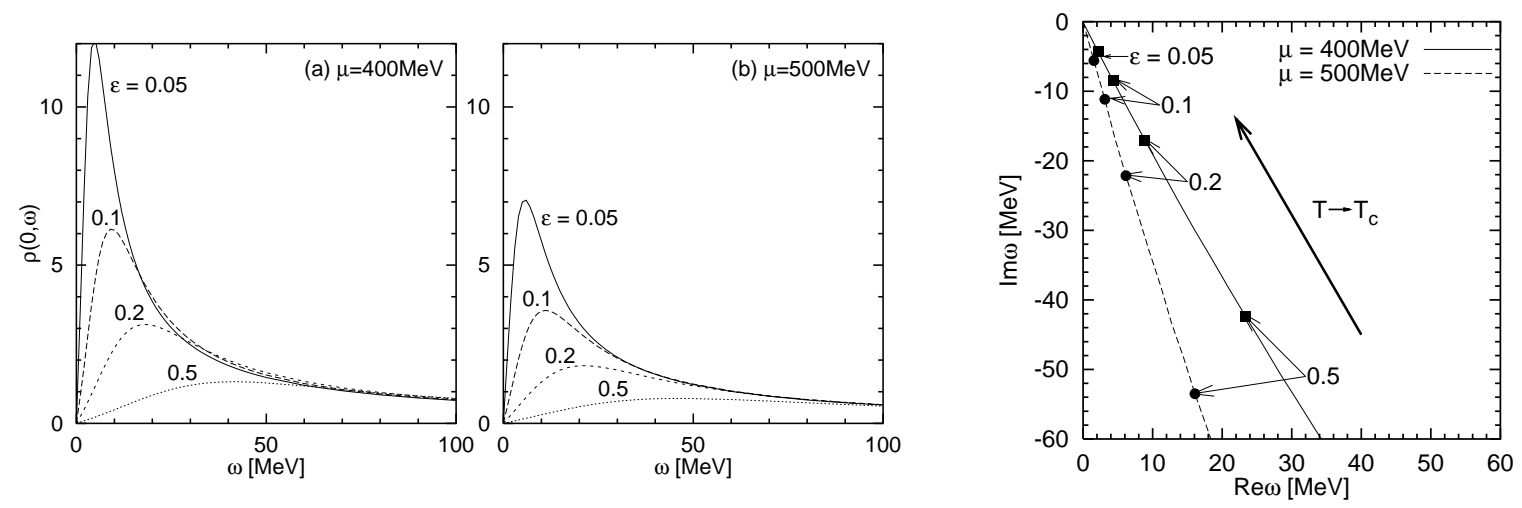

Figure 3. The spectral function for the pair field at $T>T_{c}$ with $\epsilon \equiv\left(T-T_{c}\right) / T_{c}=$ $0.05,0.1,0.2$ and 0.5 at $\mu=400 \mathrm{MeV}$ (a) and $\mu=500 \mathrm{MeV}$ (b).
Figure 4. The spectral function for the pair field at $T>T_{c}$ with $\epsilon \equiv(T-$ $\left.T_{c}\right) / T_{c}=0.05,0.1,0.2$ and 0.5 at $\mu=$ $400 \mathrm{MeV}$ (a) and $\mu=500 \mathrm{MeV}$ (b).

on account of the self-consistency condition for the second-order phase transition of the diquark condensate at $T=T_{c}$. This equality is the origin to lead to the various precritical phenomena for CSC. The nature of the fluctuation to be discussed below will not be altered even when the phase transition is weak first order owing to the fluctuations of the gluon fields.

The strength of the fluctuation is given by the spectral function

$\rho(\mathbf{k}, \omega)=\frac{1}{2 \pi G_{C}} \operatorname{Im} D^{R}(\mathbf{k}, \omega)$,

where $\omega>0$. The $T$ dependence of the spectral function at $\mu=400 \mathrm{MeV}$ and zero momentum transfer $(\mathbf{k}=0)$ is shown in Fig. 3(a). One can see a prominent peak moving toward the origin grows as $T$ is lowered toward $T_{c}$ as indicated by the arrow in Fig. 1. In the experimental point of view, it is interesting that the peak survives at $T$ even well above $T_{c}$ with $\epsilon \equiv\left(T-T_{c}\right) / T_{c} \sim 0.2$. This means that the precritical region of CSC is one to two order larger in the unit of $T_{c}$ than that in the usual electric superconductors of metals. The result for $\mu=500 \mathrm{MeV}$ is shown in Fig. 3(b); one sees that although the growth of the peak becomes relatively moderate, the qualitative features do not change.

\section{SOFT MODE AND PARACONDUCTIVITY}

The existence of the peak with the narrow width suggests an existence of a well-defined collective mode as an elementary excitation in the Wigner phase. This can be examined by searching possible poles of the response function. The existence of a pole $\omega=\omega(k)$ with given $k$ means that the pair field $\Delta_{i n d}(k, \omega(k))$ can be created even with an infinitesimal external field $\Delta_{e x}^{*}$ as seen from Eq. (2);i.e., the system admits spontaneous excitation of a collective mode with the dispersion relation $\omega=\omega(k)$. (One should notice that the gap equation Eq. (3) implies that $z=0$ is a solution at $T=T_{c}$, i.e., there exists a zero mode at the critical point.) The singular behavior seen in the spectral function near the critical 

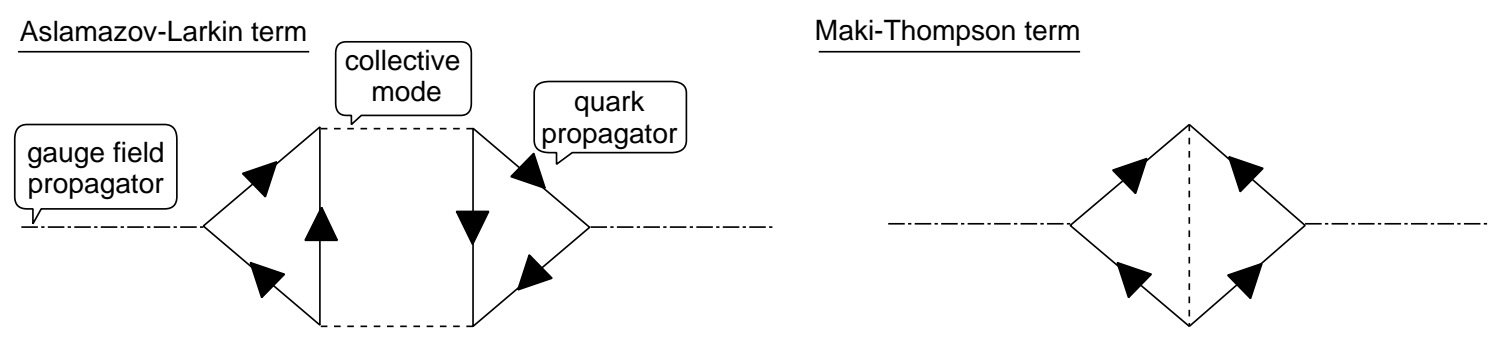

Figure 5. Diagrams which contribute paraconductivity. The left and right figures correspond to the Aslamazov-Larkin and Maki-Tompson terms, respectively.

point in Fig. 3 is caused by the presence of the zero mode. A numerical calculation shows that there indeed exists a pole in the lower half plane at $T>T_{c}$. Fig. 4 shows how the pole moves as $T$ is lowered toward $T_{c}$ for $\mu=400$ and $500 \mathrm{MeV}$ at $\mathbf{k}=0$. One sees that the pole approaches the origin as $T$ is lowered toward $T_{c}$. Such a mode whose energy tends to vanish as the system approaches the critical point of a phase transition is called a soft mode. Another characteristic feature of the soft mode is that the absolute value of the imaginary part $\omega_{i}$ of the pole is larger than that of the real part $\omega_{r}$ for both $\mu$. This feature may have an important implication for an effective equation describing the dynamical phase transition for CSC: The dynamical behavior of the order parameter near $T_{c}$ is well described by a non-linear diffusion equation like the time-dependent GinzburgLandau equation.

Such a soft mode gives rise to anomalous behaviors of several transport coefficients; for example, an anomalous excess of the electric conductivity above $T_{c}$ which is known as paraconductivity (PC)[国. The diagrammatic contributions to PC are known as AslamazovLarkin and Maki-Tompson terms, which are shown in Fig. 5. It is worth emphasizing that these diagrams can be regarded as a modification of the self-energy of gauge fields, i.e., the gluons and the photons, which decay into dilepton pairs. This implies that photons and hence dileptons from the system can carry some information of the fluctuation of CS. We also notice that the pairing fluctuations can give rise to a pseudo-gap phenomena known in high- $T_{c}$ superconductivity of copper-oxides. It would be intriguing to pursue possible similarity of CSC and high- $T_{c}$ superconductivity. These are under investigations.

\section{REFERENCES}

1. For recent reviews, see K. Rajagopal and F. Wilczek, Chapter 35 in the Festschrift in honor of B. L. Ioffe, "At the Frontier of Particle Physics / Handbook of QCD", M. Shifman, ed., (World Scientific); M. Alford, Ann. Rev. Nucl. Part. Sci. 51, 131 (2001).

2. T. Tsuneto, "Superconductivity and superfluidity", Cambridge University Press, 1998.

3. M. Kitazawa, T. Koide, T. Kunihiro and Y. Nemoto, Phys. Rev. D65, 091504(R) (2002).

4. L. G. Aslamazov and A. I. Larkin, Sov. Phys. Solid State 10, 875 (1968); K. Maki, Prog. Theor. Phys. 40, 193 (1968); R. S. Thompson, Phys. Rev. B1, 327 (1970). 